\title{
Will Education Post-2015 Move Us toward Environmental Sustainability?
}

\author{
Hikaru Komatsu, Jeremy Rappleye and Iveta Silova
}

Every year the problems are getting worse. We are at the limits. If I may use a strong word I would say that we are at the limits of suicide.

POPE FRANCIS, SPEAKING AT THE COP21 PARIS CLIMATE CHANGE CONFERENCE

(2015)

Introduction: Education at the Limits ${ }^{1}$

Developing sustainable societies is now recognised as the foremost challenge of the 21st century. In 2015, United Nations member states ratified the Sustainable Development Goals (SDGs), a set of targets that purported to place the global community on the path toward a sustainable future. The SDGs sought to extend the previous Millennium Development Goals (MDGs), but also incorporate new domains that, since the 199os, had been widely recognised as pressing global challenges. Foremost among these new challenges is the stark reality of climate change. The 2015 ratification of the SDG s coincided with the historic UN Climate Change Conference, held in Paris (COP21) - the global community's most ambitious attempt to date to keep global warming below the $2^{\circ} \mathrm{C}$ threshold. Failure to do so, the Paris Agreement warned, would lead to 'catastrophic' consequences.

The SDGs feature targets for education, with SDG 4 explicitly seeking to achieve 'quality education for all' through a combination of increasing access to education, raising quality, equalising existing inequalities (e.g., in gender), and ensuring that 'all learners acquire knowledge and skills needed to promote sustainable development, including among others through education for sustainable development and sustainable lifestyles, human rights, gender equality, promotion of a culture of peace and non-violence, global citizenship, and appreciation of cultural diversity and of culture's contribution to sustainable 
development' (UNGA, 2015b, p. 17; see also Appendix 1). SDG 4 is also promoted as essential for achieving sustainability.

Nonetheless, some scholars and practitioners have been critical about the purported effectiveness of SDG 4 for the achievement of sustainability. First, it has been pointed out that the SDG 4 'emphasises education in terms of its potential economic and social benefits' rather than its environmental consequences (Sterling, 2015, 2016). Second, the overall logic of the SDGs is that 'the goals depend on each other - but no one has specified exactly how' (Nilsson, Griggs, \& Visbeck, 2016, p. 320). This becomes even more problematic when considering that national 'policymakers lack tools to identify which interactions are the most important to tackle, and evidence to show how particular interventions and policies help or hinder progress towards the goals'. That is, 'if countries ignore the overlaps [between SDGs] and simply start trying to tick off targets one by one, they risk perverse outcomes' (Nilsson et al., 2016, pp. $\left.3^{20}-321\right)$. Third, and in support of the point made above, extensive analyses of major United Nations and World Bank reports revealed 'weak coverage of linkages between education and SDGs 12-15' (Vladimirova \& Le Blanc, 2016; see also Le Blanc, 2015). Those latter four goals are directly related to the environment and target sustainable consumption and production, climate change, oceans and marine resources, and terrestrial ecosystems.

The Agenda 2030 framework refers to 'interlinkages' between the 17 SDGs (UNGA, 2015b, pp. 2, 31, 34). However, those interlinkages remain implicit and poorly articulated in SDG statements (Nilsson et al., 2016). The United Nations agencies, such as UNESCO, and other international organisations seek to encourage interlinkages between education and other SDGs, at least to some extent. However, our argument herein is that when interactions between different SDGs are earnestly considered by these actors, they are nearly always imagined as exclusively positive, that is, reflecting the assumption that improving existing forms of education will positively impact environmental sustainability.

However, we find this core assumption surprising and highly problematic, given that the world is the most 'educated' it has ever been and yet the nearest to environmentalbreakdown(Randers,2012;Turner,2012;UNGA,2015b;Rappleye\& Komatsu, in press; Silova, Komatsu, \& Rappleye, 2018). At the heart of the problem is a de facto endorsement of and a continued reliance on modern mass schooling, one rooted in the 'modernist Western paradigm' (Sterling, Dawson, \& Warwick, 2018, p. 5; Silova, Komatsu, \& Rappleye, 2019). Most international development efforts thus far, including education initiatives associated with the MDGs and SDGs, have consistently prioritised Western education models that focus on economic growth, technocratic determinism, human 
exceptionalism, and liberal individualism over environmental concerns. Yet, for decades now, many scholars have underscored that education in its current form is a central part of the environmental problem (e.g., Schumacher, 1973; Bowers, 1995, 2002; Orr, 2004, 2009). Orr (2009, p. 176) wrote:

Education has long been a part of the problem, turning out graduates who were clueless about the way the world works as a physical system or why that knowledge was important to their lives and careers, while at the same time promoting knowledge of the sort that has fuelled the destruction of ecologies and undermined human prospects.

What is problematic is not simply that the United Nations does not accept the fact that education in its current form is a central part of the environmental crisis. Rather the problem is that this possibility has not even been seriously considered by the world's most powerful 'development' actors, as we review in detail in the pages that follow.

Against this backdrop, the objective of this chapter is threefold. First, we confirm that potentially negative interactions between education and other SDGs are largely missed and/or dismissed in SDG-related policy and analytical work by major international organisations, such as UNESCO, the OECD, and the World Bank. This dismissal of negative interactions between education and other SDGs means that the current education paradigm remains unquestioned, resulting in the continued prioritisation of economic growth and social equity over the environment. Second, we present empirical evidence of the possibility that promoting education based on the current paradigm can have negative impacts on the achievement of other SDGs. For this purpose, we use one example: analysing the potentially negative interaction between promoting education access and quality (SDG 4) and alleviating climate change (SDG 13). Third, we then shift to spotlight a missing component within the existing analyses of the current education paradigm - culture. We suggest that culture, which encodes our attitudes and values, strongly affects our environmental impacts on the Earth. ${ }^{2}$ Through these interlocking three steps, we underscore the pressing need to reflect deeply on the consequences of the current education paradigm, one rooted in the 'modernist Western paradigm' for climate change (Sterling et al., 2018). Our purpose is to initiate a different sort of conversation than the one that currently surrounds SDG 4: one that helps researchers, practitioners, and policymakers alike imagine something beyond the current education paradigm and gives the next generation a chance to shift off our current trajectory of environmental catastrophe. 

Unquestioned?

Within the existing literature, potentially negative interactions between education and other SDGs are largely overlooked or dismissed, leaving the current education paradigm unquestioned. Here we analyse the SDG targets themselves and their interpretation as presented in major reports published by UnESCO, the OECD, and the World Bank. Although implementation of the SDGs is officially envisaged as being led by national policymakers and planners, research has consistently shown that these international organisations have strong influence on the contours of national policymaking (e.g., Edwards, 2012; Verger, Edwards, \& Altinyelken, 2014; Auld, Rappleye, \& Morris, 2019). In some cases, the influences can be decisive for developing countries that have limited policymaking capacity or funding (Rahman \& Quadir, 2018; Rappleye \& Un, 2018; Auld et al., 2019). This section confirms that the negative interactions between education and other SDGs go unquestioned, resulting in a de facto prioritisation of business-as-usual in education (i.e., continued prioritisation of economic growth and social equity over the environment).

\subsection{SDG Targets}

SDG 4 comprises seven targets (4.1-4.7) and three means of implementation (4.a, 4.b, and 4.c) (for details, see Appendix 1). The first six targets (4.1-4.6) primarily focus on the improvement of access and quality of education. As such, these six targets do not require reconsideration of current approaches and policy preferences, but instead call for more thoroughgoing implementation. This dominance of the current education paradigm in the SDGs seems to imply that contemporary problems are largely the result of incomplete implementation, rather than one cause of our current condition.

This strong belief in continuity with past approaches is clearly expressed in the United Nations' announcement of the SDGs (UNGA, 2015b). The Preamble states:

We recognize that eradicating poverty in all its forms and dimensions, including extreme poverty, is the greatest global challenge and an indispensable requirement for sustainable development. (UNGA, 2015b, p. 1)

It then continues:

The 17 Sustainable Development Goals and 169 targets which we are announcing today ... seek to build on the Millennium Development Goals 
and complete what they did not achieve. They seek to realize the human rights of all and to achieve gender equality and the empowerment of all women and girls. (UNGA, 2015 b, p. 1)

Here we see the SDGs are viewed as an extension of the MDGs, and the effort is to expand the current development and education paradigm via more thorough implementation, rather than affecting a change of course.

In contrast with the first six, the last target (4.7) suggests that the current education paradigm itself has negative interactions with other SDGs. It includes the phrase 'education for sustainable development and sustainable life styles' (UNGA, 2015b, p. 17). For some this might suggest an approach to education that goes beyond the current education paradigm, questioning the role of education in contributing to and reproducing the economic growth logic. Unfortunately, it is left unclear whether this reading is possible due to the simplicity and ambiguity of the wording of Target 4.7. We shall return to this issue later when we analyse reports published by international organisations.

Beyond SDG 4, several targets in other SDGs do mention education (e.g., Targets $3.7,8.6,12.8$, and 13.3). For this reason, it could be argued that different SDGs and associated targets must be viewed as not independent but interlocking. Yet what is striking here is that education is always deemed to be in the service of progress toward other SDGs. For example, SDG Target 13.3 states:

Improve education, awareness-raising and human and institutional capacity on climate change mitigation, adaptation, impact reduction and early warning. (UNGA, 2015b, p. 23)

Improvement of education is automatically assumed to lead to mitigation of the damages caused by climate change. We do not object to this possibility. But we find it problematic that the SDGs do not address the possibility that education in its current form is not the solution but instead one cause of climate change and other related environmental problems.

\section{$2.2 \quad$ UNESCO}

UNESCO has recently published several major reports focussing on the SDGS. In reviewing these reports here, we sought to understand how much UNESCO recognises potentially negative interactions between education and other SDGs. We found that negative interactions are almost never discussed.

Education 2030: Incheon Declaration and Framework for Action for the Implementation of Sustainable Development Goal 4 discusses the interaction between education and other SDGs as follows: 
Education can accelerate progress towards the achievement of all of the SDGs and therefore should be part of the strategies to achieve each of them. (WEF, 2015, p. 24)

In contrast to this wholly positive but terse appraisal, a subsequent 2017 report entitled Education for Sustainable Development Goals: Learning Objectives elaborates a bit more deeply. The report admits that 'not all kinds of education support sustainable development' (UNESCO, 2017c, p. 7), perhaps a sign of UNESCO's awareness of the need to pursue a different educational paradigm. However, the report unexpectedly continues that the education for achieving sustainability (i.e., Education for Sustainable Development, ESD) is 'now wellestablished', describing it as follows:

ESD is holistic and transformational education that addresses learning content and outcomes, pedagogy and the learning environment. Thus, ESD does not only integrate contents such as climate change, poverty and sustainable consumption into the curriculum; it also creates interactive, learner-centred teaching and learning settings. What ESD requires is a shift from teaching to learning. (UNESCO, 2017c, p. 7)

Disappointingly, these 'interactive learner-centred teaching and learning settings' are what the current global education paradigm already valorises (and has valorised for the past several decades). The report assumes that such education practices based on the current paradigm 'make possible the development of the key competencies needed for promoting sustainable development' (UNESCO, 2017c, p. 7). It is not difficult to find similar examples dotted around other UNESCO reports. For example, the UN Decade of Education for Sustainable Development (DESD) Report states:

ESD is influencing learning pedagogies and advancing approaches that help learners to ask questions, analyse, think critically and make decisions in collaboration with others. ... Participatory learning processes, critical thinking and problem-based learning are proving particularly conducive to ESD. (UNESCO, 2014d, p. 30)

Here, ESD and learner-centred pedagogy are depicted as virtually synonymous. Furthermore, UNESCO has suggested that the methodologies outlined in the DESD report should become part of the mechanisms to assess the progress toward Target 4.7 (UIS, 2018e, p. 37), underscoring that UnESCO shows little intent to reflect more deeply on the current education paradigm. 
What does this 'current education paradigm' entail? One way to describe it is by referring to UNESCO's explanation of key competencies for achieving the SDGs:

As societies around the world struggle to keep pace with the progress of technology and globalization, they encounter many new challenges. These include increasing complexity and uncertainty. ... A rapidly proliferating amount of information is available to them. All these conditions require creative and self-organized action because the complexity of the situation surpasses basic problem-solving processes that go strictly according to plan. People must learn to understand the complex world in which they live. They need to be able to collaborate, speak up and act for positive change. ... We can call these people 'sustainability citizens'. (UNESCO, 2017c, p. 10)

There are two implicit assumptions in this statement. First, 'sustainability citizens' must be able to process and analyse much information to understand the world around them. Second, 'sustainability citizens' must organise their own behaviours rationally based on their own understanding, rather than react to and interact with the environments around them. In the following paragraphs, we discuss the OECD, but here it is worth highlighting how closely its vision of 'key competencies' aligns with that of UNESCO:

Key competencies assume a mental autonomy, which involves an active and reflective approach to life. They call not only for abstract thinking and self-reflection, but also for distancing oneself from the socializing process. ... This means being self-initiating, self-correcting, and self-evaluating rather than dependent on others to frame the problems, initiate adjustments, or determine whether things are going acceptably well. (OECD, 200o, p. 13)

Here the valorisation of abstract thought and independence is striking: all problems are ultimately problems of the self. Both this and UnEsco's 'sustainability citizens' conceptualisation turn out to be almost indistinguishable from the ideal 'man' of the Western Enlightenment, which emphasises the use of rationality and individual autonomy to improve the conditions of human beings (Duignan, 2018). The Western Enlightenment provides the conceptual foundations for progressive education (Thomas, 2012). Progressive education in turn is the origin and driver of student-centred pedagogy, in which students are expected to rationally organise their actions, learn from the outcomes of 
those actions, and eventually achieve success through self-directed projects (for a critique of this approach, see Komatsu \& Rappleye, 2017c).

In contrast with the UNESCO reports reviewed here, the web pages of UNEsco go one step further in a critical direction (UNESCO, 2018a, 2018c). These pages address the necessity of 'reorienting education' to 'transform society' mainly through SDG Target 4.7 and to 'help people develop knowledge, skills, values and behaviours needed for sustainable development' (UNESCO, 2018a). This statement implies that the current education paradigm needs to be reoriented. However, nowhere in these web pages are the problems of the current education paradigm explicitly discussed, probably due to space limitations.

To our knowledge, the only material describing explicitly the problem of the current education paradigm in any substantial depth is the $2016 \mathrm{Global}$ Education Monitoring Report (GEMR). GEMRs are editorially independent of UNESCO and cannot be used to argue for UNESCO's official opinion. Still, they may give us some sense of the thinking among those working in close proximity to the organisation. In its first chapter, the 2016 GEMR explicitly admits that 'the Millennium Development Goals failed to ensure environmental sustainability'. Citing the final MDG review, the GEMR points out that 'the cost to future generations of environmental damage during development was not evaluated, as it was commonly believed that countries could grow now and clean up later' (UNESCO, 2016b, p. 5). Indeed, in 1997, about three years before the MDGs were launched, the signatories of the United Nations Framework Convention on Climate Change agreed to the Kyoto Protocol introducing legally binding emission reduction targets for developed countries. Not only did the Kyoto Protocol fail to find its way into the MDGs, but global $\mathrm{CO}_{2}$ emissions have been continuously rising since 1997 .

Recognising the failure of the MDGs on this front, the 2016 GEMR tried to propose fundamental changes to the current education paradigm. Chapter 1 appears to reject the high-modern assumptions of the past, that is, the faith that science, technology, human rationality, and individual creativity will make a transition to a sustainable society. Instead, the chapter directs attention beyond the usual 'modern solutions', highlighting divergent development trajectories and 'pre-modern' or 'non-modern' conceptual resources. Indeed, the chapter depicts several countries outside the European high-modern cultural orbit as being successful in reconciling environmental sustainability and human well-being:

Countries struggle to find balance between human development and sustainable practices. Some, including Cuba, Georgia, the Republic of 
Moldova and Sri Lanka, have begun to find it, managing to keep production and consumption within sustainable bounds. (UNESCO, 2016b, p. 22)

The report goes on to list other successful countries including Jamaica, Colombia, the Dominican Republic, Indonesia, and the Philippines (UNESCO, 2016b, p. 23, figure 1.2B).

The report contrasts these cases with the relatively unsuccessful countries of Europe, North America, and the Asian Tigers, all areas that have led the world in economic growth over the past three decades:

The countries with the largest ecological footprints are mostly in Europe and Northern America. Countries that have experienced rapid increases in education, health and living standards, including the Republic of Korea and Singapore, have seen their ecological footprint nearly double as domestic consumption has expanded. (UNESCO, 2016b, p. 22)

Particularly in its critique of South Korea and Singapore, this report seems to signal a radical departure from its previous views of education. Instead of a positive evaluation of the 'development success' of East Asian economic systems through education (e.g., OECD, 2011; World Bank, 2018e), we find this more negative evaluation precisely because the environment is considered to be equally as important as economics and education.

\subsection{The OECD}

Reviewing the official OECD web page about the SDGs and two major reports recently published there, Better Policies for 2030: An OECD Action Plan on the Sustainable Development Goals (OECD, 2016a) and Measuring Distance to the SDG Targets: An Assessment of Where OECD Countries Stand (OECD, 2017a), we found no explicit interpretation of the SDGs. Instead, the OECD exclusively focusses on more thorough implementation of the existing education paradigm. The OECD web page states that 'the OECD supports the United Nations in ensuring the success of the 2030 Agenda for Sustainable Development by bringing together its existing knowledge, and its unique tools and experience' (OECD, 2018b).

The OECD evinces virtually no reflection on the effectiveness of SDGs vis-àvis the environment. Instead, it suggests that its contribution to the achievement of SDGs will be providing 'measures and systems for monitoring' the progress (OECD, 2018b). Similarly, Better Policies for 2030 states that the OECD aims to 'support countries as they identify where they currently stand in relation to the SDGs' (OECD, 2016a, p. 3). The primary purpose of Measuring 
Distance is monitoring. The report 'uses the latest information on various indicators available in OECD databases to establish countries' distance from individual targets' (OECD, 2017a, p. 1).

Interestingly, a new report published by the OECD, entitled The Future of Education and Skills: Education 2030, implies there are potentially negative impacts of education on society and the environment. The report states that 'the rapid advance of science and technology may widen inequities, exacerbate social fragmentation and accelerate resource depletion' (OECD, 2018a, p. 3 ). However, the forms of education the OECD envisages as necessary to combat these negative impacts are exactly those based on the current education paradigm (i.e., emphasis on basic literacy and numeracy skills, student-centred pedagogy directed toward cognitive 'gains', individualised instruction accelerated with technology, etc.). The report asserts that future education needs to have 'a personalised learning environment that supports and motivates each student to nurture his or her passions, make connections between different learning experiences and opportunities, and design their own learning projects and processes in collaboration with others', as well as building a solid foundation that includes 'literacy and numeracy' and 'digital literacy and data literacy' (OECD, 2018a, p. 4). Here the commitment to the Western Enlightenment paradigm seems obvious: personalised, passionate, experience-based, and technologically mediated.

\subsection{The World Bank}

As with our analysis of the OECD, we reviewed the official World Bank web pages that discuss the SDGs and three major reports recently published by the Bank. The World Bank simply interprets the SDGs as already aligned with its existing goals. The web page states that 'the SDGs are aligned with the World Bank Group's twin goals of ending extreme poverty and boosting shared prosperity' (World Bank, 2018c). The same interpretation of the SDGs is echoed in other reports (World Bank, 2016, 2017a). For example, the Bank's 2015/2016 Global Monitoring Report states that 'the two sets of goals [the SDGs and the World Bank's existing goals] can be seen as very similar' (World Bank, 2016, p. 99).

Considering this interpretation of the SDGs, it makes sense that the World Bank repeatedly declares its intention to improve access to and quality of education without finding it necessary to contemplate possible negative impacts of the current form of education on the environment (e.g., World Bank, 2016, p. $8 ; 2017$ a, p. 21). It seems obvious that the World Bank has not seriously reflected on the current education paradigm but sees it as universally valid in both space and time. For example, the 2018 World Development Report emphasises South 
Korea's 'successful economic development' through education (World Bank, 2018d, p. xi) but mentions none of the environmental caveats that were raised in the 2016 GEMR.

The absence of serious reflection on the current education paradigm is evident both in the overall conceptualisation of the role of education in the SDGs and its operationalisation by major international agencies in the field of education, including UNESCO, OECD, and the World Bank. Such unreflective acceptance of the current education paradigm has resulted in a de facto endorsement of business-as-usual in education, in other words, continued prioritisation of economic growth and social equity over the environment, further reinforced by a narrow range of data used to monitor achievement of the SDGs. Perhaps this also explains why major reports by the OECD (2017a) and the World Bank (2018e) focus on how their data (e.g., Programme for International Student Assessment [PISA]) will contribute to monitoring achievement of SDGs, ensuring value-for-money in implementing predetermined policy decisions, and identifying the most efficacious mechanisms for financing and implementing the existing education paradigm, instead of radically reevaluating its impact vis-à-vis environmental sustainability.

Potentially Negative Interaction between Education and the Environment

We have thus far demonstrated that official statements by leading international organisations largely lack contemplation about possible negative interactions between current forms of education and the environment. This section uses empirical data to highlight the presence of such negative interactions, focussing on the relations between improvement of education access and quality, on the one hand, and climate change, on the other.

\subsection{Data and Methods}

We examine the relationships between the lower-secondary completion rate and $\mathrm{CO}_{2}$ emissions per capita, as well as between the percentage of students having a 'fixed level of proficiency' in literacy and numeracy skills according to the OECD's definition and $\mathrm{CO}_{2}$ emissions per capita for various countries. The OECD defines the 'fixed level of proficiency' in literacy and numeracy skills as being identical to Level 2 or higher in PISA (Hanushek \& Woessmann, 2015; OECD, 2017a). Our analysis excluded data from major oil exporters (e.g., Bahrain, Iran, Kuwait, Oman, Qatar, Saudi Arabia, Trinidad and Tobago, and United Arab Emirates) because the $\mathrm{CO}_{2}$ emissions of these countries did not 
follow the general relationship between education and $\mathrm{CO}_{2}$ emissions (i.e., $\mathrm{CO}_{2}$ emissions for these countries were much higher than expected from the education indicators).

Although SDG 4 has a large number of indicators, we decided to use the lower-secondary enrolment rate and the percentage of students having a fixed level of proficiency as proxy indicators for the general level of education of any given population. Indeed, these indicators are widely used or recommended in major reports concerning the SDGs (e.g., UNESCO, 2016b; World Bank, 2016; OECD, 2017a). We are fully aware that these indicators do not fully represent or cover the whole scope of SDG 4. But the two proxy indicators selected represent the current education paradigm and its twofold aim of enhancing access as well as quality, as also captured in SDG 4, and are appropriate to the primary purpose of our analysis. That purpose is to detect the presence of interactions between education and the environment, not to assess the total impact of education on the environment. Our focus on the abovementioned indicators is primarily due to the availability of data. However, the fact that so much data are available for these indicators suggests the magnitude of attention and political power they have.

Our analysis of the relationships between education and $\mathrm{CO}_{2}$ emissions per capita is based on simple correlation analysis. The relationships are assessed using the Pearson's correlation coefficient $(r)$. Since an $r$ value is highly affected by outliers, we calculate $95 \%$ confidence intervals (CI) to examine the stability of the correlation using the bootstrapping method (Diadonis \& Efron, 1983; Komatsu \& Rappleye, 2017c). We do not conduct statistical testing of any relationships and differences because such an approach is quite often deeply misleading, particularly in the field of education. ${ }^{3}$

Since we use simple correlation analysis, we do not attempt to evaluate a direct impact of education on $\mathrm{CO}_{2}$ emissions. We are rather interested in understanding the impact of accelerating the implementation of the dominant educational paradigm, including the improvement of education access, on $\mathrm{CO}_{2}$ emissions. Our focus in the analysis is whether or not there are countries with a high enrolment rate and percentage of students with a fixed level of proficiency, but at the same time with low $\mathrm{CO}_{2}$ emissions per capita. If there are no countries satisfying these conditions, education in its current form would potentially have negative interactions with the environment.

\subsection{Results and Discussion}

Weobserved positive correlations between the lower-secondary completion rate and per capita $\mathrm{CO}_{2}$ emissions, with $r$ being $.749(\mathrm{CI}=[.649 ; .828])$ (Figure 14.1a) and between the percentage of students with the fixed level of proficiency 


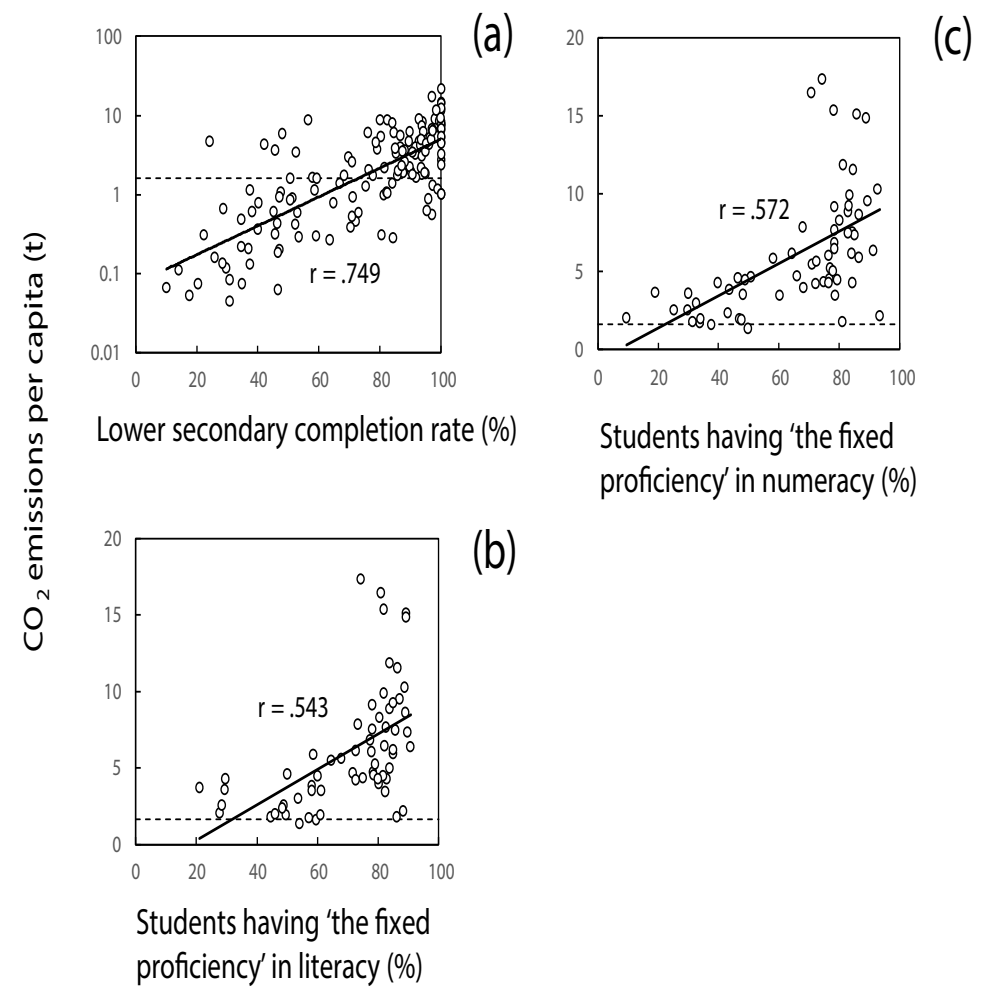

FIGURE 14.1 Relationship of (a) the lower-secondary completion rate with $\mathrm{CO}_{2}$ emissions per capita. Relationships between the ratios of 15 -year-old students with (b) basic literacy and (c) numeracy and $\mathrm{CO}_{2}$ emissions per capita. A dotted line denotes the $\mathrm{CO}_{2}$ emission per capita in 205 o in the IPCC scenario leading to 1.3 to $2.1^{\circ} \mathrm{C}$ temperature increases (van Vuuren et al., 2011). Data for the lower-secondary completion rate were derived from the World Bank Open Data (World Bank, 2018d). Data for the ratios of students with basic literacy and numeracy were derived from PISA 2015 (OECD, 2016c)

and per capita $\mathrm{CO}_{2}$ emissions with $r$ being $.543(\mathrm{CI}=[.418 ; .667])$ for reading and $.572(\mathrm{CI}=[.442 ; .708])$ for math (Figures 14.1b and 14.1c). That is, countries with 'better' education tended to have more detrimental impacts on climate change. These positive correlations would probably be even more pronounced if we considered international trades in foodstuffs and industrial products. That is, rich countries tend to have 'better' education and also externalise their $\mathrm{CO}_{2}$ emissions through international trade. For example, some of the $\mathrm{CO}_{2}$ emissions generated by American consumption are attributed to China, which serves as one of many 'factories' for the production of American goods and therefore absorbs much of the pollution associated with it (Komatsu, Rappleye, \& Silova, 2019b). 
What is important from the climate change perspective is that few countries with 'good' education satisfied the Intergovernmental Panel on Climate Change (IPCC) target to limit the global mean temperature increase to less than $2^{\circ} \mathrm{C}$ relative to preindustrial levels (van Vuuren et al., 2011; Houghton, 2015). If we assume only the current global population and no future population growth, ${ }^{4}$ per capita $\mathrm{CO}_{2}$ emissions need to be reduced to $1.61 \mathrm{t}$ (dotted lines in Figure 14.1) or lower by 2050 to meet the target. ${ }^{5}$ Only seven countries among the 61 with a high lower-secondary completion rate (>90\%) had per capita $\mathrm{CO}_{2}$ emissions lower than 1.61 $\mathrm{t}$ (Figure 14.1a). Furthermore, no countries with a high percentage of students with the fixed proficiency (>70\%) had per capita $\mathrm{CO}_{2}$ emissions lower than 1.61 $\mathrm{t}$ (Figures 14.1b and 14.1c).

It is true that improvement in education (particularly education of girls and women) usually leads to lower fertility rates, resulting in the alleviation of population growth (Martin, 1995; Osili and Long, 2008) and this, in turn, may lead to a reduction in $\mathrm{CO}_{2}$ emissions. However, we assumed no change in the world population between the present and 2050 in the prior discussion to account for such possible effects. Yet our data suggest that the current per capita $\mathrm{CO}_{2}$ emissions are too high to be offset by the reduction in the population growth rate through education alone.

Advocates for the SDGs might further argue that our analysis ignores the interrelated nature of the SDGs and their targets, and mistakenly assesses the impact of selected targets of SDGS on the environment. This argument is understandable, but our analysis did not intend to assess the impact of education on the environment as a whole. Rather, the primary purpose is to highlight the presence of potentially negative interactions between targets in SDG 4 and the environment, which are largely ignored in the discussion about SDGs. Our analysis thus should be viewed as a necessary first step to understand how SDG 4 targets are (or are not) interrelated with other targets.

In fact, what we show here is not without precedent. It has been widely known for over two decades that improvement in education accompanies the rise of economic output and $\mathrm{CO}_{2}$ emissions (Hotz-Eakin \& Selden, 1995). ${ }^{6}$ Sceptical readers might argue that although this might be the case for developing countries, developed countries are now reducing $\mathrm{CO}_{2}$ emissions. It is true that several developed countries were successful in reducing $\mathrm{CO}_{2}$ emissions per economic output, which might be related to the improvement of education. However, most of these countries have been and still are unsuccessful in reducing the total $\mathrm{CO}_{2}$ emissions (Raupach et al., 2007; Jackson, 2009), ${ }^{7}$ suggesting that improvement in education quality would not lead to the alleviation of climate change. Our findings in this section thus complement these previous studies in environmental economics and sciences. 
What then is the novelty of our analysis? It lies in connecting the issue of negative interactions between education and the environment with the current debates over the SDGs. As we underscored in the previous section, virtually no reports by international organisations mention such negative interactions. Recognising interactions (including negative ones) among different SDGs is a prerequisite for effective policymaking in the future.

What Is Missing from the Current Education Paradigm?

The above two sections suggest that at least some parts of current education practices have negative interactions with the environment. It is thus unreasonable to continue promoting reforms based on the belief that a thoroughgoing expansion and achievement of the current education paradigm will lead to sustainability, particularly environmental sustainability. Our previous analysis suggested that the dominant education paradigm assumes, true to Western Enlightenment logic, that if people are equipped with the skills to reason and the knowledge about the problems they face, they will act to solve the problems. Against this backdrop, this section suggests the possibility that knowledge and skills alone would not be sufficient to achieve environmental sustainability. Instead, we suggest that culture, which encodes our attitudes and values, strongly affects human impacts on climate.

\subsection{Data and Methods}

We examine the relationships between awareness and risk perception of climate change and $\mathrm{CO}_{2}$ emissions for various countries. Data for awareness and risk perception of climate change were derived from the Gallup Poll 20072008 (Pugliese \& Ray, 2009). The Gallup Poll included data for the percentages of people who were aware of climate change and who viewed climate change as a personal threat. Concerning the first issue, respondents were asked, 'How much do you know about global warming or climate change?' and allowed to select one option among the four: (1) have not heard of it, (2) know something about it, (3) know a great deal about it, and (4) don't know/refused. The percentage of those who selected the second or third options was used in the analysis. Concerning the second issue raised by the poll, respondents were asked, 'How serious of a threat is global warming to you and your family?' and allowed to select one option among the four: (1) very/somewhat serious, (2) not very/not serious at all serious, (3) don't know/refused, and (4) not aware. The percentage of those who selected the first option was used in the analysis. 
We then examine the relationship between one of Hofstede's cultural dimensions (i.e., the individualism vs. collectivism dimension; Hofstede, Hofstede, \& Minkov, 2010). The degree of individualism was represented by the individualism score derived from the 2010 version dataset for Hofstede's cultural dimensions (Hofstede et al., 2010). This dataset assesses characteristics of national culture for various countries and has been used widely (e.g., Park, Russell, \& Lee, 2007; Peng \& Lin, 2009; Onel \& Mukherjee, 2014). An individualism score, which represents the degree of individualism for a given country, ranges between $o$ and 100 with higher values indicating more individualistic.

In our analysis, we use data only for countries having a sufficiently long life expectancy (i.e., no less than 75.5 years). This threshold of 75.5 years allowed us to include all the core members of the OECD and to eliminate potential arguments about the trade-offs between long life and environmental sustainability. This treatment is necessary because countries with lower awareness and risk perception of climate change may lack basic conditions for life and have low per capita $\mathrm{CO}_{2}$ emissions. The threshold of 75.5 years' life expectancy thus captures countries with large contributions to the global ecological footprint (EF): $61.7 \%$ of global EF relative to their population, which is $39.2 \%$ of the world's total (see Komatsu, Rappleye, \& Silova, 2019a). ${ }^{8}$

\subsection{Results and Discussion}

We did not observe a negative correlation between awareness and $\mathrm{CO}_{2}$ emissions (Figure 14.2a). Rather, the correlation was positive $(r=.625$ with CI being $[.451 ; .773])$. Similarly, we did not observe a clear negative correlation between risk perception and $\mathrm{CO}_{2}$ emissions $(r=-.07$ with $\mathrm{CI}$ being $[-.417 ; .241]$, Figure $14.2 \mathrm{~b})$. That is, countries whose people are aware of climate change and perceive the potential risks of climate change did not always have lower per capita $\mathrm{CO}_{2}$ emissions.

These findings suggest that knowledge alone would not be sufficient to achieve environmental sustainability. It is true that many previous studies found that people with more knowledge of environmental science tend to have higher concern for environmental problems (Meyer, 2015; Chankrajang \& Muttarak, 2017). Joel Westheimer addresses a similar point concerning civic education in Chapter 13. But our findings call into question whether this higher concern will lead people to successfully reduce their actual environmental impacts.

Figure 14.2c shows the relationship between individualism scores and $\mathrm{CO}_{2}$ emissions. The correlation was clear and positive $(r=.556$ with $\mathrm{CI}$ being $[.213 ; .774])$. That is, countries where individualism is stronger tended to have higher per capita $\mathrm{CO}_{2}$ emissions. Inversely, countries where interdependence 

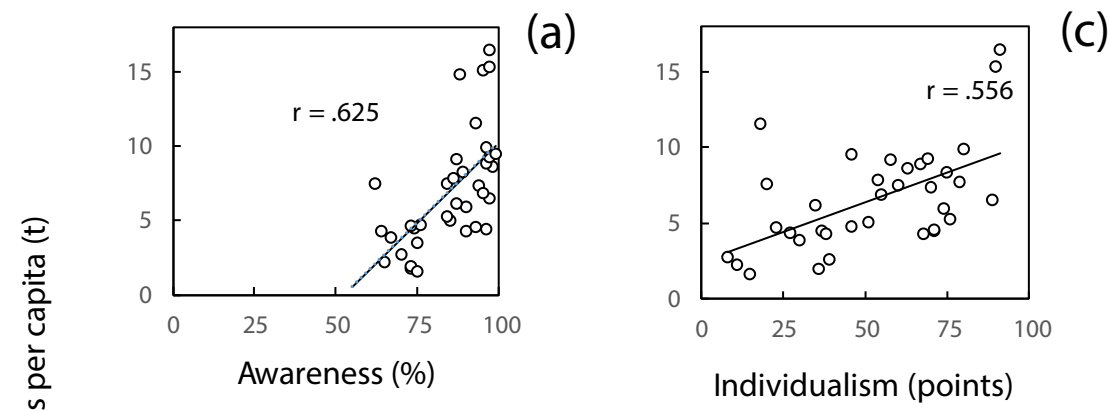

(c)

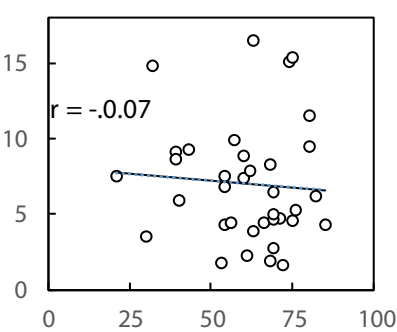

(b)

\section{Risk perception (\%)}

FIGURE 14.2 Relationships of (a) awareness and (b) risk perception of climate change and individualism scores with $\mathrm{CO}_{2}$ emissions per capita. This analysis used data only for countries whose life expectancy is no less than 75.5 years. Data were derived from the Gallup Poll (Pugliese \& Ray, 2009), the World Bank Open Data (World Bank, 2018d), and Hofstede et al. (2010)

(collectivism) is stronger tended to have lower per capita $\mathrm{CO}_{2}$ emissions. We are fully aware that correlation does not prove causation. Our exploratory argument needs further examination and elaboration. Still these results suggest the possibility that culture affects actual human impacts on climate.

Our findings align with previous studies in environmental psychology (Arnocky, Stroink, \& DeCicco, 2007; Adger et al., 2013; Chuang, Xie, \& Liu, 2016). This last-cited study reported that the interdependent self, which is more prevalent in interdependent (collectivist) cultures, is more effective in controlling one's own desire for the sake of collective social benefit and consequently willing to engage in pro-environmental behaviour. In addition, Arnocky et al. (2007) reported that interdependent selves cooperated more effectively with others than independent selves under hypothetical conditions of resource constraints. This finding also supports the idea that interdependent selves control (regulate) their behaviour more effectively than independent selves when they are faced with environmental problems or dilemmas. The novelty of our findings is thus that a specific dimension of culture concerning the concept of selfhood could be a major factor explaining not only 
within-country but between-country variations in people's environmental attitudes. ${ }^{9}$ More importantly, our analysis revealed that cultural dimensions are strongly related to actual human impacts on the Earth. To date, this point has not been sufficiently addressed in environmental psychology (e.g., Schultz, 2001; Gifford, 2014; Chuang et al., 2016). Readers interested in details of a large study we conducted are referred to Komatsu et al. (2019a).

We then asked whether or not the variation in per capita $\mathrm{CO}_{2}$ emissions with the cultural dimension is large enough to affect the possibility of meeting the IPCC target. If per capita emissions for the world population's equal those for Costa Rica (whose per capita $\mathrm{CO}_{2}$ emissions and individualism score were low), the global $\mathrm{CO}_{2}$ emission is estimated to be $3.23 \mathrm{Gt}$ [gigatonnes $=10^{10}$ tonnes] carbon, which is equivalent to $11.84 \mathrm{Gt} \mathrm{CO}_{2}$ (see Figure 14.3). This value is $33 \%$ of current global emissions and almost equivalent to the global emission needed by 2050 to meet the IPCC $2^{\circ} \mathrm{C}$ target (3.19 Gt carbon). What then happens if we assume that the per capita $\mathrm{CO}_{2}$ emissions for the world population equals those for the mean per capita $\mathrm{CO}_{2}$ emissions for individualistic societies (i.e., the mean per capita $\mathrm{CO}_{2}$ emissions for countries having individualism scores higher than 75$)$ ? All those countries are located in Europe or North America (Figure 14.4). The global $\mathrm{CO}_{2}$ emission under this assumption is estimated as $19.7 \mathrm{Gt}$ carbon. That value is close to the global emission in $205^{\circ}$

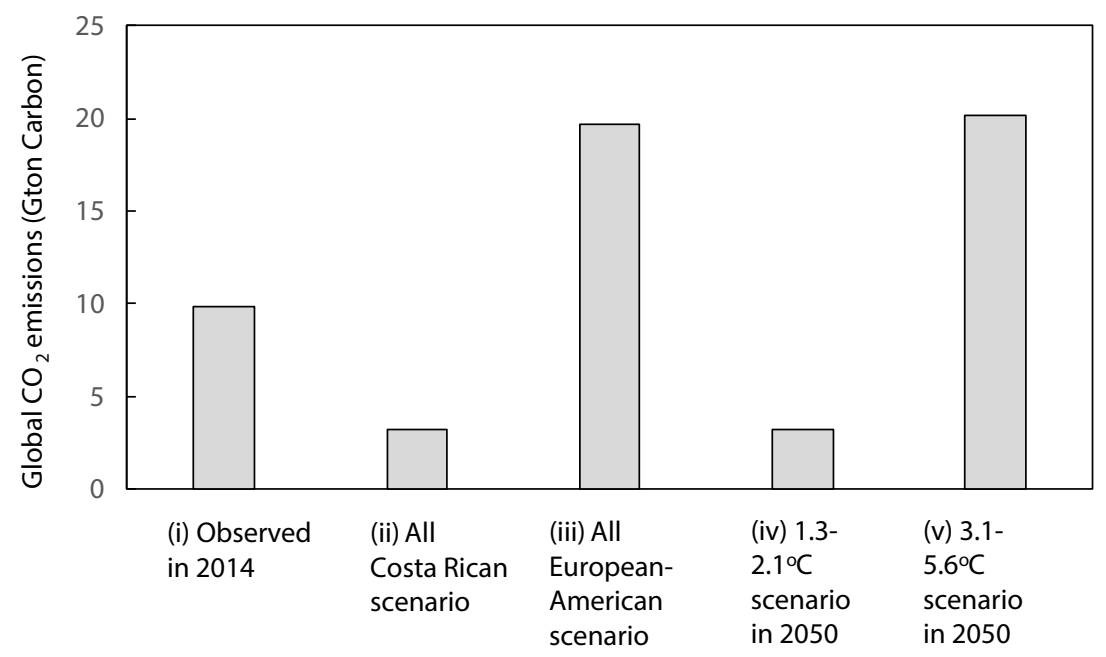

FIGURE 14.3 Global $\mathrm{CO}_{2}$ emissions for different cases. (i) actual emissions in 2014; emissions assuming that $\mathrm{CO}_{2}$ emissions for the world population equal (ii) those of Costa Rica; (iii) those of Europe and America; and emissions in 2050 in the IPCC scenarios leading to (iv) 1.3 to 2.1 and (v) 3.1 to $5.6^{\circ} \mathrm{C}$ temperature increases, respectively. Data for the IPCC scenarios were derived from van Vuuren et al. (2011) and Meinshausen et al. (2011) 
(a) America

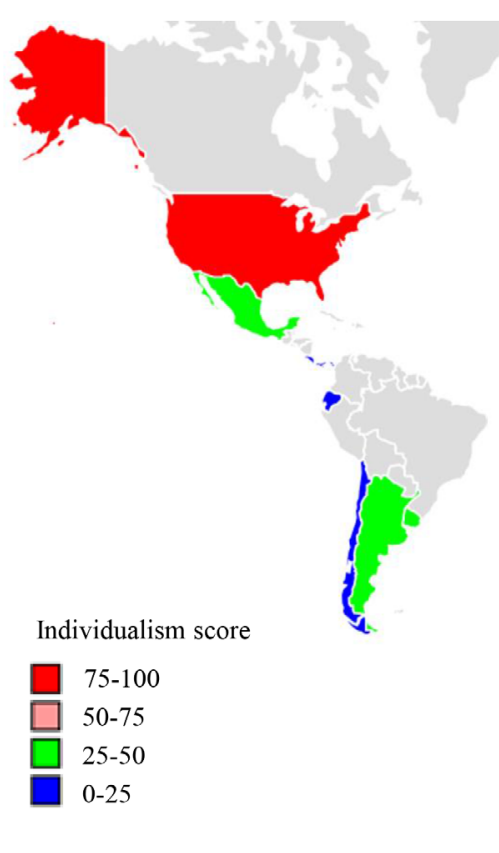

(b) Europe

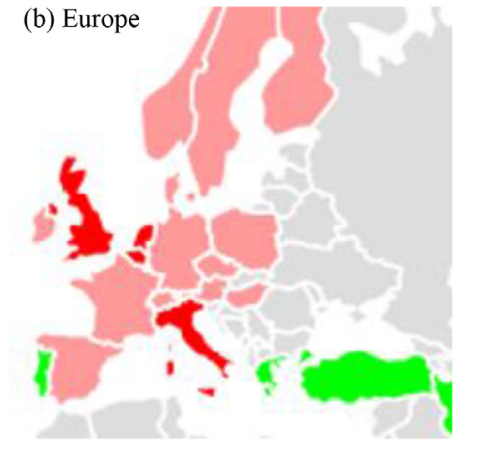

(c) Asia \&

Oceania

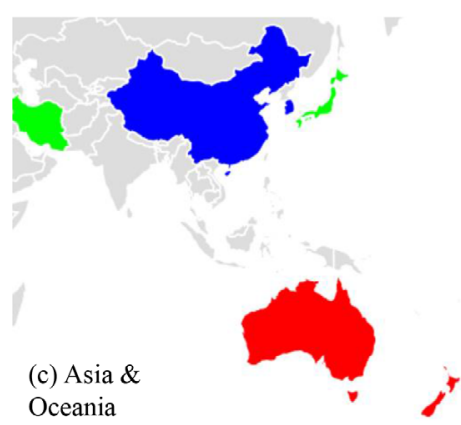

FIGURE 14.4 Countries classified by the individualism score. Countries lacking individualism scores and whose life expectancy is short (i.e., $<75.5$ years) are not coloured. Data for individualism scores were derived from Hofstede et al. (2010)

in the scenario assuming a 3.1 to $5.6^{\circ} \mathrm{C}$ temperature increase (20.2 Gt carbon). If the correlation in Figure 14.2c does represent causality, the effect of cultural factors would be quite large from an environmental standpoint.

The results described above have serious implications for SDG 4. Although SDG 4 and related discussions occasionally mention the need to change behaviours and underlying cultural patterns, a much stronger emphasis on knowledge and skills is everywhere apparent. It is true that ESD is assumed to play the role of changing people's behaviours and culture. However, the current conceptualisation of ESD narrowly promotes one form of education, which is anchored in Western modernist schooling and privileges human exceptionalism and liberal individualism over other values. Yet such an approach promotes independent rather than interdependent selfhood (Rappleye \& Komatsu, 2017; Rappleye et al., 2020). This notion of independent selfhood is a very explicit goal of leading international organisations, as reviewed above. That makes sense when considering that student-centred education has its origin in the West where independent selfhood is the de facto assumption (Thomas, 2012, Siedentop, 2014). If interdependent selfhood is one key for achieving sustainability, it is questionable whether ESD as currently practised, emphasising 
forms of education characterised by independence, rationality, and the atomised 'self', will contribute to our collective recognition of and progress toward environmental sustainability. We understand this is a provocative suggestion, but more provocations are necessary to avoid business-as-usual as the planet moves closer to catastrophic consequences.

\section{$5 \quad$ Conclusions}

We began this chapter by confirming that negative interactions between education and other SDGs are largely dismissed in SDG statements and their interpretations by major international organisations. This dismissal means that the current education paradigm remains unquestioned, resulting in the continued prioritisation of economic growth and social equity over the environment. We then suggested the presence of such negative interactions by taking an example of the interaction between education and climate change. Finally, we pointed out that cultural dimensions concerning the concept of selfhood might be a factor strongly affecting human environmental impacts on the Earth, although culture and the concept of selfhood are not even recognised within the current education paradigm, let alone central to current debates on education and environmental sustainability.

Based on these findings, we make the following three recommendations for scholars, practitioners, and policymakers:

- Policymakers and practitioners should be duly cautious about the effectiveness of ESD as currently proposed.

- Scholars and practitioners need to become aware of the deeper assumptions underlying different education practices.

- Scholars and international organisations should rethink the relevance of universal scales of sustainability.

The following describes each of these recommendations as invitations for future research and debate.

\subsection{Caution about the Effectiveness of ESD}

Although ESD has been proposed as one of the central mechanisms of achieving sustainability by major actors, including international organisations, our findings suggest that national policymakers and practitioners should be duly cautious about the effectiveness of the ESD in its current form. While it is true that the current approach to ESD initiates some movement in the direction 
of sustainability, the underpinning assumptions about subjectivity and selfhood of the ESD approach are largely indistinguishable from, and too easily fall into complementarity with, the currently dominant education paradigm. Those who question the effectiveness of the currently proposed ESD are not only critical scholars, such as Bowers (1995, 2002), Orr (2004, 2009), Sterling (2016), and Sterling et al. (2018), but in fact thinkers who once subscribed to the approach themselves (UNESCO, 2014d, p. 184).

\subsection{Becoming Aware of Different Assumptions Underlying Education Practices}

Because the effectiveness of ESD as currently practised is uncertain, scholars and practitioners need to look for educational practices based on different assumptions than those of the 'modernist Western paradigm' (Sterling et al., 2018). One promising means to do so is to look to non-Western countries and indigenous cultures where the current education paradigm has not been fully internalised. Indeed, the IPCC's October 2018 Special Report on Climate Change explicitly mentions that 'education, information, and community approaches, including those informed by indigenous knowledge and local knowledge, can accelerate the wide scale behavior changes consistent with adapting to and limiting global warming' (IPCC, 2018: section D.5.6). Yet, while there is some awareness of the importance of non-Western education practices among some policy stakeholders, it is disappointing that major international organisations, such as the World Bank and the OECD, are now seeking to replace remaining elements of indigenous systems in non-Western countries with those based on the current education paradigm, often through the creation of international large-scale assessments and related competencybased curricular changes (Takayama, 2008; Gorur, 2016; Addey, 2017; Auld et al., 2019). For a fuller discussion of these ideas, see Silova et al. (2018) and the subsequent response by Vickers (2018).

We believe that some critical distance from Western modes of thinking and education - a particular cultural arrangement, not a universal phenomenon is a crucial step for locating alternatives beyond the Western education paradigm as we face the climate change catastrophe. As this chapter has illustrated, the education paradigm promoted by major international agencies reflects not only the economic growth logic of these organisations but also the 'subjectivity' projects they try to enact globally, e.g., a focus on abstract thinking (cognition alone); and the reduction of 'society' to an agglomeration of atomised (neo)liberal individuals competing for rapidly shrinking resources on Earth. 
We seek to open space for questioning whether such approaches are conducive to sustainable lifestyles, not necessarily provide answers. We are requesting that scholars and policymakers reimagine education on a much wider scale, considering alternatives beyond the Western education paradigm that can contribute to the collective effort to think in new ways.

One very promising, school-based alternative is the Japanese Food for Education programme (Komatsu \& Rappleye, 2018). At the same time, experimental attempts to go beyond the current education paradigm are also available in Western contexts, for example, the overall curriculum approach of Schumacher College in the United Kingdom (Sterling et al., 2018). ${ }^{10}$ Similarly, many indigenous practices aim to reintegrate alternative ways of coexisting with nature and the Earth within the modern school curriculum (Masuku van Damme \& Neluvhalani, 2004; Glasson et al., 2010; Shukla, Barkman, \& Patel, 2017). These initiatives work under the assumption that the individual is not independent but fully embedded in social and natural networks (Sterling et al., 2018), offering alternative resources for thinking about sustainability of environment and culture.

There is an urgent need to set up an arena to exchange information about such experiments. Several academic journals (e.g., Journal of Transformative Education) have been playing a limited role in this regard. Yet this means the exchange is still restricted to very narrow academic circles, usually working on the periphery of mainstream research and facing considerable obstacles in a Western-dominated linguistic and publishing world. This work has now a far more urgent priority than monitoring education access and quality in pursuit of the 'modernist Western paradigm'. Is there any reason that international organisations could not help facilitate such information exchange?

\subsection{Rethinking the Relevance of Universal Scales of Sustainability}

We are highly sceptical about the effectiveness of establishing a 'universal scale' to measure progress to sustainability, although several international organisations seem to be intent on doing so. Using scales in such a way implicitly assumes that the current world lacks something important and therefore it should be achieved by progress. This assumption, which is in fact a globally dominant worldview, and its policy impacts, are exactly what led us to our current world: increasingly dire environmental challenges met only with continued economic growth, technological innovations, and extension of 'progress' targets (Uchiyama, 2010). Readers might argue that the most critical contemporary problem is runaway capitalism rather than our ontology (i.e., fundamental building blocks of a worldview). Our position is that runaway capitalism is one consequence of our current ontology. We cannot thus solve the problem without rewriting our ontology. 
If we still believe in the effectiveness of having a scale, it should rather be organised to measure how much we have forgotten. This is exactly opposite to the direction in which the OECD and other international agencies are moving. Humans potentially have (and perhaps originally had) various relationships, including religious and spiritual ones, with nature. But modern people tend to forget this fact and see nature as merely a standing reserve of commodities, such as water, timber, and minerals. Like others, we too see excessive consumption as correlated with what has been forgotten; capitalist excesses and catastrophic consumption run freest where the amnesia about other ways of being is most acute (Mita, 2018). Thus, relevant scales should be redirected to 'measuring' ontological and psychological dimensions. This is clearly not an easy task, but fortunately various subfields of psychology, including environmental, social, and cultural psychology, have already accumulated useful knowledge and preliminary tools to guide us in the task (e.g., Frantz et al., 2005; Arnocky et al., 2007; Stroink \& DeCicco, 2011; Amerigo, Aragones, \& Garcia, 2012; Gifford, 2014; Chuang et al., 2016). Connecting approaches found in cultural psychology with education policies and actual embodied practice are an urgent task for education scholars and international organisations alike.

We opened this chapter with the unprecedented pessimism of the Pope over the current 'suicide' trajectory. 'People no longer seem to believe in a happy future', he wrote in the more extended Encyclical Letter on the environmental problem published six months before the Paris Climate Talks, suggesting, 'If we want to bring about deep change, we need to realize that certain mindsets really do influence our behavior. Our efforts at education will be inadequate and ineffectual unless we strive to promote a new way of thinking about human beings, life, society, and our relationship with nature' (Francis, 2015, p. 157). And what precisely is to be the crux of that education-led renewal, that crucial shift in 'mindset'? The move away from individualism: 'Isolated individuals can lose their ability and freedom to escape the utilitarian mindset. ... Social problems must be addressed by community networks and not simply the sum of individual good deeds' (Francis, 2015, p. 160). 'Networks' mean precisely relations over atomised entities. How might education foster those? What new pedagogies are needed? Would those education systems that never had the Westernturned-modern ideology of individualism so deeply entrenched have resources for us to learn from (Dumont, 1986; Bellah et al., 1985; Taylor, 1989)?

'There needs to be a distinctive way of looking at things, a way of thinking, policies, an educational programme, a lifestyle and a spirituality which together generate resistance to the assault of the technocratic paradigm', wrote Pope Francis. 'To seek only a technical remedy to each environmental problem which comes up is to separate what in reality [is] interconnected' (Francis, 2015, p. 84). In this chapter, we have expressed our pessimism over current 
SDG 4 discussions that explore mere technical solutions and neglect changes in mindset. We deplore the almost total disregard for environmental issues. More constructively, we suggest that we can learn from sources that sit outside the Western tradition precisely because the deepest issues are cultural. The Pope's passion and problématique are laudable, but we would depart from him over where to go looking for 'new ways of thinking'. Becoming aware of those alternatives and then connecting to them helps mitigate the pessimism wrought by the illusion of isolation.

\section{Notes}

1 All authors contributed equally to this chapter.

2 This perspective has been repeatedly put forth by leading philosophers, historians, sociologists, and others over the past several decades (e.g., White, 1967; Maki, 1977[2003]; Naess, 1989; see also Komatsu \& Rappleye, 2017b). However, very few education scholars and practitioners show any awareness of this discussion unfolding over the past 50 years.

3 The primary reason for not conducting statistical testing is that even very weak relationships or very small differences can be statistically significant if sample sizes are sufficiently large. For example, a relationship having $r$ of .oo62o is statistically significant if the sample size exceeds 100,000. This problem has already been identified for decades (Berkson, 1938; Bakan, 1966; Carver, 1978) and echoed by many contemporary researchers (Thompson, 1996, 2002; Nuzzo, 2014; Komatsu \& Rappleye, 2017a). For this reason, many researchers have shifted to reporting confidence intervals and effect sizes (e.g., $r$, Spearman's $r h o$, Cohen's $d$, and Glass' delta) to avoid the problems of statistical significance (Johnson, 1999; Thompson, 2002; Komatsu, Shinohara, \& Otsuki, 2015).

4 In truth, this is an impossibly optimistic starting point. Currently the projection for global population by 2050 is $9-11$ billion (depending on the model utilised). This is an increase of 2-4 billion since 2012 .

5 Even these difficult-to-reach estimates are likely overly optimistic. A recent study published in Nature (Resplandy et al., 2018) argues that previous research had underestimated ocean heat uptake due to methodological constraints and that, in fact, to achieve the Paris target of $2^{\circ} \mathrm{C}$ warming, emissions will need to be reduced by more than previously thought. That is, the original estimates underestimated the extent and speed of the coming catastrophe.

6 Education is not only a cause but a result of economic growth. Furthermore, the contribution of education to economic growth is not decisive as commonly advocated by proponents of extreme human capital theory (Komatsu \& Rappleye, 2017a). 
7 This is also the case when using a more comprehensive indicator (i.e., Ecological Footprint of Consumption) instead of $\mathrm{CO}_{2}$ emissions (York, Rosa, \& Dietz, 2004, 2009; Dietz, Rosa, \& York, 2012). Not only developing countries, but developed countries are unsuccessful in reducing Ecological Footprint of Consumption.

8 In our analysis, we do not use multiple linear regression analysis, although it is widely used particularly by social scientists. Using that method would have included untested assumptions in the analysis (see Komatsu, et al., 2019a). One problematic assumption of multiple linear regression analyses is that all independent variables affect the dependent variable in a linear way. But those relationships are quite often nonlinear (e.g., Komatsu et al., 2014). Another problematic assumption is that different independent variables affect the dependent variable in an additive manner. Different factors actually quite often affect dependent variables in a multiplicative manner, as widely acknowledged by natural scientists (e.g., Komatsu et al., 2014). Owing to these problems, we do not use multiple linear regression analysis in this study.

9 In particular, a specific concept of self interacts with its specific social environments, e.g., institutions, practices, products, and others (Markus \& Kitayama, 2010; Komatsu et al., 2019a). The relationship between individualism scores and EF would stem not only from differences in patterns of personal consumption, but from social institutional arrangements. For example, collectivistic societies may be more prone to establishing institutions to promote pro-environmental behaviour (UNESCO, 2016b, p. 27).

10 Nevertheless, it is dangerous and counterproductive to seek to identify 'best practices' for sustainability without awareness of the complex contexts within which such practices occur and without attunement to different assumptions underlying such education practices. One major reason for this is fundamental differences in the underpinning assumptions of self, others, time, and space among societies (e.g., Markus \& Kitayama, 1991, 2010; Nisbett, 2003; Rappleye \& Komatsu, 2016, 2017, in press; Rappleye et al., 2020; Silova, 2019). It may be more productive to learn from practices of other societies to consciously realise the underlying assumptions of one's own. 